${ }^{1} \mathrm{~S}$ I ljaz, ${ }^{2}$ Jerbeek, ${ }^{3} \mathrm{~A}$ Ojajaarvi, ${ }^{3} \mathrm{~K}$ Neuvonen. ${ }^{1} \mathrm{FIOH}$, Kuopio, Finland; ${ }^{2}$ The Finnish Institute of Occupational Health, Finland; ${ }^{3} \mathrm{FIOH}$, Kuopio, Finland

\subsection{6/oemed-2013-101717.354}

Objective Night work and shift work are among the most prevalent occupational exposures. It is postulated that night work can result in a higher risk of breast cancer. There is uncertainty in evidence from previous research to implicate night work in causing breast cancer. We did a systematic review to assess the strength of association between exposure to night shift work and breast cancer incidence.

Methods Multiple databases and non-electronic sources were systematically searched to identify case control and cohort studies involving females in night shift work. The comparison was non-shift or day work and the outcome was incident breast cancer. We assessed studies for risk of bias using a content specific piloted checklist on 10 domains of interest. We performed random effects meta-analysis and meta-regressions of study-specific incremental relative risks to determine the risk of cancer associated with a 5 year and 300 night shift increases in exposure. Sensitivity analyses were performed to test model assumptions. Results We included 16 studies (12 case control and four cohorts). Ten studies were conducted in Western Europe, four in USA and two in China. Almost half of the studies were on nurses. None of the studies were at a low risk of bias however five were at a moderate risk of bias. Studies with appropriate exposure assessment were lacking, with only one measuring exposure in an objective way prospectively. Twelve studies (nine case controls and three cohorts) provided data for the random effects meta-regression of dose response using generalised least square estimates.

Conclusions Several new studies have become available since the last review in 2008. However, exposure assessment in existing studies is still far from optimal. Results of the meta-analysis will be presented at the conference.

\section{NIGHT SHIFT WORK AND PROSTATE CANCER RISK IN A POPULATION-BASED CASE-CONTROL STUDY IN SPAIN}

${ }^{1} \mathrm{M}$ K Kogevinas, ${ }^{1}$ Papantoniou, ${ }^{2}$ Gómez Acebo, ${ }^{3}$ Merino Salas, ${ }^{4}$ Peiro, ${ }^{5}$ Perez, ${ }^{6}$ Alguacil. ${ }^{1}$ CREAL-Centre for Research in Environmental Epidemiology, Barcelona, Spain; ${ }^{2}$ University of Cantabria, Santander, Santander, Spain; ${ }^{3}$ Servicio de Urología del Hospital Universitario San Cecili, Granada, Spain; ${ }^{4}$ Centro Superior de investigación en salud pública, Valencia; Spain; ${ }^{5}$ National Centre of Epidemiology-Carlos III, Madrid, Spain; ${ }^{6}$ Universidad Huelva, Huelva, Spain

\subsection{6/oemed-2013-101717.355}

Objectives Recent epidemiologic and animal data indicate that night work may increase the risk of cancer and specifically breast cancer. There is limited evidence on other hormone related cancers. We evaluated prostate cancer risk and night shift work in a population based case-control study in Spain, the MCC-Spain.

Methods Incident prostate cancer cases $(\mathrm{n}=1117)$ and randomly selected population controls $(n=1165)$ were enrolled in 7 regions of Spain. Lifetime occupational history including details on shift work, and information on lifestyle factors were assessed by face-to-face interviews. We estimated the risk of different shift profiles using unconditional logistic regression models adjusting for a wide range of potential confounders.

Results Among the 2282 subjects, 12\% reported having ever worked in permanent night shift and $17 \%$ in rotating night shift for $\geq 1$ year. Having ever worked in night work (including permanent and rotating) was associated with a small and non-significant increased risk for prostate cancer (Odds Ratio (OR) 1.15, 95\% CI 0.94-1.39) compared to day workers, after adjusting for age, centre, educational level and family history. This small increase was due to an increase in the ORs for rotating night shift workers $(\mathrm{OR}=1.26,95 \% \mathrm{CI} 0.99-1.63)$. ORs were slightly increased for workers with more than 30 years permanent night work $(\mathrm{OR}=1.22)$ and those in rotating shift $(\mathrm{OR}=1.34)$. Results will be presented for different shift profiles and lifetime cumulative exposure.

Conclusions In this large population based study we did not find an overall clear increase in prostate cancer risk associated with permanent or rotating night work. The analysis is ongoing and, at the conference, results will be presented for more detailed exposure classifications of night shift.

(Additional authors: G. Castaño, CREAL, Barcelona, M. Rivas-Fresno, Hospital Cabueñes, Asturias)

\section{Session: 26. Exposure assessment methods II}

\section{NATIONAL ESTIMATES OF THE PREVALENCE OF OCCUPATIONAL CARCINOGEN EXPOSURE}

${ }^{1} \mathrm{~L}$ Fritschi, ${ }^{1}$ Carey, ${ }^{2}$ Driscoll, ${ }^{1}$ Reid, ${ }^{1} S$ Peters, ${ }^{3}$ Benke, ${ }^{3} \mathrm{D}$ Glass. ${ }^{1}$ University of Western Australia, Perth, Australia; ${ }^{2}$ University of Sydney, Sydney, Australia; ' ${ }^{3}$ Monash University, Melbourne, Australia

\subsection{6/oemed-2013-101717.356}

Objectives To estimate the burden of occupationally-related cancer, the prevalence of occupational exposure to carcinogens is necessary, and this information is not available in most countries.

Methods We surveyed 5023 Australian workers aged 18 to 65 to determine the prevalence of exposure to 38 occupational carcinogens. We developed job specific modules (JSMs) for 57 jobs and industries with questions on the determinants of exposure to the 38 agents and the use of control measures. The answers to the questions were linked by algorithms to assessments of probability and level of exposure within a web-based application (OccIDEAS). Interviewers called randomly-selected phone numbers and asked consenting participants about their current job. If that job had any likelihood of exposure to any of the 38 agents, the interviewer administered the most relevant JSM for that job. The algorithms were then run and the automatic assessments were reviewed by the project co-ordinator, with more complex assessments being referred to occupational hygienists for review.

Results About half of the subjects $(n=2498)$ were in a job with potential exposure. The most common jobs were construction workers, drivers and health workers. $40 \%$ of subjects were exposed to at least one carcinogen, with the most common exposures being solar UV, diesel exhaust and environmental tobacco smoke. We will also present profiles of exposure by industry. For example, almost all carpenters are exposed to wood dust at a high level, while about $80 \%$ of painters are exposed to wood dust, half of them at at a high level and half at a low level.

Conclusions The prevalence of exposure to carcinogens at work is quite high and a survey such as ours is useful in pinpointing areas where control of exposure is not adequate. Our findings can be used in calculating the burden of disease from occupational exposures. 